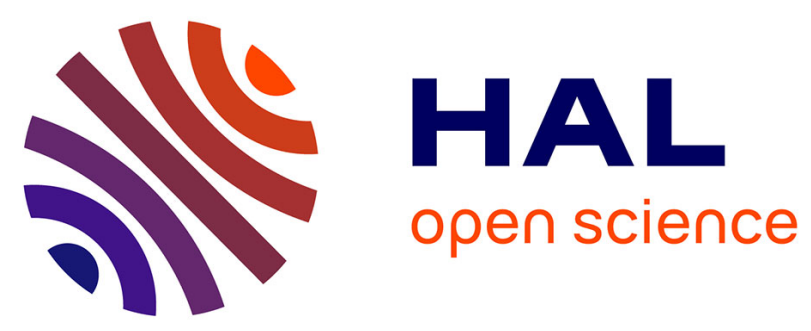

\title{
Transcriptome analysis of genes involved in the response of a pollinator fig wasp to volatile organic compounds from its host figs
}

Jiqing Zeng, Hui Yu, Finn Kjellberg

\section{- To cite this version:}

Jiqing Zeng, Hui Yu, Finn Kjellberg. Transcriptome analysis of genes involved in the response of a pollinator fig wasp to volatile organic compounds from its host figs. Acta Oecologica, 2018, 90, pp.91-98. 10.1016/j.actao.2018.01.003 . hal-02333163

\section{HAL Id: hal-02333163 \\ https://hal.science/hal-02333163}

Submitted on 25 Oct 2019

HAL is a multi-disciplinary open access archive for the deposit and dissemination of scientific research documents, whether they are published or not. The documents may come from teaching and research institutions in France or abroad, or from public or private research centers.
L'archive ouverte pluridisciplinaire HAL, est destinée au dépôt et à la diffusion de documents scientifiques de niveau recherche, publiés ou non, émanant des établissements d'enseignement et de recherche français ou étrangers, des laboratoires publics ou privés. 
Transcriptome analysis of genes involved in the response of a pollinator fig wasp to volatile organic compounds from its host figs

Jiqing Zeng ${ }^{\mathrm{a}, \mathrm{b}}$, Hui $\mathrm{Yu}^{\mathrm{a}, \mathrm{c}, *}$, Finn Kjellberg ${ }^{\mathrm{d}}$

${ }^{a}$ Guangdong Provincial Key Laboratory of Digital Botanical Garden, South China Botanical Garden, The Chinese Academy of Sciences, Guangzhou 510650, PR China ${ }^{\mathrm{b}}$ Key Laboratory of South China Agricultural Plant Molecular Analysis and Genetic Improvement, South China Botanical Garden, Chinese Academy of Sciences, Guangzhou 510650, PR China

Key Laboratory of Plant Resource Conservation and Sustainable Utilization, South China Botanical Garden, The Chinese Academy of Sciences, Guangzhou 510650, PR China

CEFE, UMR 5175, CNRS - Université de Montpellier - Université Paul-Valéry Montpellier EPHE, 1919 Route de Mende, F-34293 Montpellier Cédex 5, France

Keywords: Ficus hirta, Fig, Fig wasp, Fig-fig wasp recognition, Mutualism, Transcriptome. 


\begin{abstract}
The mutualism of figs and their pollinating fig wasps is widely regarded as a model for coevolved mutualism. A high degree of host specificity is ensured by female wasps only being attracted by their specific fig tree species through the volatile organic compounds (VOCs) released by the figs when they are ready to be pollinated. However, very little is known about the molecular mechanisms underlying the production of VOCs and how pollinators respond to these VOCs. Here we present transcriptome sequencing data from VOC-treated fig wasps and control fig wasps. Using Illumina paired-end sequencing, approximately $6.47 \mathrm{Gbp}$ and 6.48 Gbp high quality reads were generated for fig wasps that had been exposed or not to VOCs of their host fig. After read trimming, the de novo assembly of both types of reads produced 58,192 unigenes with an average length of $817 \mathrm{bp}$. Then functional annotation and GO enrichment analysis was performed by aligning all-unigenes with public protein databases including NR, SwissProt, and KEGG. Differentially expressed genes (DEGs) were investigated using the RPKM method. Overall, 16 up-regulated genes and 13 down-regulated genes were identified. We further performed GO enrichment and metabolic pathway enrichment analyses. One gene involved in the synoptic vesicle cycle and two genes coding for odorant binding proteins (OBP) are likely to have potential impacts on the response of fig wasps to the VOCs emitted by their host figs. This is the first transcriptome sequencing of a fig wasp in the presence of VOCs of its host figs using the next-generation sequencing technology. Our studies suggest that the expression of some genes in the olfactory neural system of the fig wasps is affected by the VOCs released from the figs. This suggests the presence of a dynamic molecular system of detection and hence response to host plant VOCs. As such our findings provide indications for further mechanistic studies on the fig-fig wasp interactions.
\end{abstract}

\title{
1. Introduction
}

Fig trees (Ficus spp. Moraceae), have a largely pan-tropical distribution, and form one of the largest genera of woody plants, with more than 750 species (Berg, 2003). The interactions between fig trees and fig wasps provide one of the classic examples of pollination mutualism and present a model for investigating co-evolution (Cruaud et al., 2010). Fig trees produce figs which are urn-shaped inflorescences, lined on the inside by hundreds or thousands of tiny female flowers, each of which can produce one fig wasp or one seed. Gall induction coincides with oviposition and pollination and takes place after the entry into a inflorescence of one or more foundress pollinator fig wasps.

The specificity of the relationship between a Ficus species and its pollinating wasps is maintained by a series of biological filters: volatile organic compounds (VOCs), ostiole (entry into the fig) structure and life history matching (Weiblen, 2002; Cook and Segar, 2010). To locate receptive figs, fig wasps mainly rely on identifying the volatile compounds the figs release, and are able to locate these figs despite the presence of other odors in the environment (Hossaert-Mckey et al., 1994; Grison-Pigé et al., 2002; Hossaert-Mckey et al., 2010). In the fig- 
fig wasp interaction system, the molecular interaction between floral smell and the fig wasps antennae is essential for the location and selection of hosts (Grison-Pigé et al., 2002; Raguso, 2008; Soler et al., 2011). The odor emitted by figs varies according to inflorescence development stage and species. To locate appropriate breeding sites, the fig wasps must accurately identify the VOCs produced by receptive figs of their host fig tree species, differentiating these VOCs from those produced by co-occurring non-host-fig species (GrisonPigé et al., 2002). Once a wasp has landed on the surface of a receptive fig, it typically brushes the surface of the fig with its antennae suggesting that tactile, gustative and olfactory cues may be involved in making the final decision to enter the fig (Gibernau et al., 1998). Moreover, inside the dark and moist inflorescences, an efficient physical and chemical sensing system is required for the fig wasps to successfully oviposit and for their offspring to mate (Weiblen, 2002). Therefore, in addition to morphological adaptations of fig wasps (van Noort and Compton, 1996), they must develop a perception mechanism of VOCs produced by the receptive inflorescences (Snyder et al., 1988; Hossaert-Mckey et al., 2010). .

Chemoreception is essential for the survival and reproduction of insects through processes such as the detection of food, predators, hosts, oviposition sites and mates (Sanchez-Gracia et al., 2009). Olfaction and taste have been reported as two major chemosensory mechanisms in insects (Vosshall and Stocker, 2007). In insects, volatile molecules are generally detected by olfactory sensory neurons (OSNs) that are located in antennal sensilla. The surface of sensilla present multiple pores, and the dendrites of OSNs are infiltrated by the sensillum lymph, which contains small, water soluble, odorant binding proteins (OBPs) and chemosensory proteins (CSPs) (Sanchez-Gracia et al., 2009). The cell membrane of OSNs also contains various receptor proteins that bind odor ligands (de Bruyne and Baker, 2008). Odor receptor genes expressed on insect OSNs are classified into three families (Benton et al., 2009; Touhara and Vosshall, 2009; Kaupp, 2010), which include odorant receptors (ORs), ionotropic receptors (IRs), and gustatory receptors (GRs) (Kwon et al., 2007). Most of the VOCs released by plants are lipid soluble small molecule compounds, which bind to the odorant binding proteins in the lymph and are transported to the olfactory receptor, causing nerve impulses that allow the insect to detect external stimuli. It has been shown that OBPs participate in the first step in odour detection (Pelosi et al., 2005). However, OBP family members are highly divergent among species of insects, and the minimum overall sequence identity between species can be as low as 17\% (Vieira and Rozas, 2011a,b). The numbers and functions of these OBP genes are variable and diverse.

Although previous studies have shown that in insects chemical signals are detected by members of multigene families that encode odorant-binding proteins (OBPs), chemosensory proteins (CSPs), olfactory receptors (ORs), gustatory receptors (GRs) and ionotropic receptors (IRs) (Pelosi and Maida, 1990), the molecular mechanism of how VOCs affect the behavior of fig wasps is unclear and little genomic information is available for this non-model group of insects. 
High-throughput RNA sequencing (RNA-Seq) using next-generation sequencing (NGS) has become a powerful technology to profile transcriptomes due to its accuracy and reproducibility (Wang and Gerstein, 2009). For fig wasps, the whole genome of a single species, Ceratosolen solmsi marchali, has been sequenced. Five GRs and forty-six OR genes were found and may be associated with the process of host specialization (Xiao et al., 2013). Ceratosolen solmsi marchali, the expression of two OBP genes (CsolOBP4 and CsolOBP5) was further confirmed using real-time quantitative PCR (Wang et al., 2014). In order to better understand the attraction mechanism of fig wasp to the VOCs, we need genomic or transcriptome studies on more fig wasps species and to identify more genes related to their chemoreception.

In this study, we investigate the response of a female wasp's transcriptome to exposure to the VOCs produced by their host tree. We surmised that, on recognizing this odor, a whole set of genes would be upregulated or downregulated in preparation for the next set of physiological and behavioural challenges facing the wasp. For instance, mobilizing carbohydrates stored in fat bodies is central to fueling flight (Beenakkers et al., 1984), and on reaching a receptive fig flight comes to an end. Narrowing in towards a receptive fig, saturation of receptors by increasing concentrations of VOCs may become a problem and could potentially stimulate higher turnover of odor-binding molecules. The wasp also needs to switch on the fig entering behavior and the set of behaviors associated with the act of oviposition. To provide preliminary elements to answer these questions, we produce a database of unigenes from VOC treated and non-treated fig wasps (Valisia javana) through Illumina transcriptome sequencing and de novo sequence assembly. This allowed us to analyze the functional composition of the transcriptome. Here we particularly focus on identifying relevant candidate genes responsible for odour detection in fig wasps, and specifically examine their expression in VOC-treated fig wasps and control wasps. We hypothesize that the match between the olfactory neural system of figs wasps and VOCs released by their host's inflorescences greatly contributes to fig-fig wasp interactions. Our studies show that the transcription of some genes in the olfactory neural system is modulated by exposure again to fig VOCs suggesting a role in the attraction mechanism. Our findings shed further light on fig-fig wasp interactions and provide a better understanding of the coevolved mutualism.

\section{Materials and methods}

Valisia javana sensu lato (super-family Chalcidoidea, family Agaonidae; Cruaud et al., 2010) is the pollinator of the dioecious shrub or small tree, Ficus hirta (family Moraceae, subgenus Ficus, section Eriosycea, sub-section Eriosycea; Berg, 2003), distributed from tropical SouthEast Asia (Indonesia) to tropical and subtropical northeast India and south China (Zhou and Gilbert, 2003).

In F. hirta, as in other functionally dioecious figs, female trees produce inflorescences containing only female flowers, which produce seeds and no wasp offspring. Male trees, in contrast, serve as hosts for fig wasps, producing 'male' inflorescences containing pollen-bearing 
male flowers and female flowers that are each capable of supporting a single developing fig wasp but no seeds.

The development of male figs can be divided into five phases (A-E; Yu and Compton, 2012). The pre-receptive A-phase comes first, with the fig containing young male and female flowers and not emitting pollinator attracting VOCs. The receptive B-phase is second, with the inflorescence emitting VOCs that attract pollinating wasps. In the three subsequent developmental phases (C-E), female flowers can contain developing pollinators. In the D-phase figs, female pollinators become adult, and mate with the males before escaping in search of Bphase figs.

\subsection{Insect material, RNA extraction and sequencing}

Eight male figs close to D-phase were collected in the South China Botanical Garden and divided equally into two tubes in the laboratory. The next morning, the fig wasps flew out of the figs and more than 50 adults were collected two tubes. Then, eight B-phase male inflorescences and eight B-phase female inflorescences were put into one tube of fig wasps (the SM sample), and the other tube of fig wasps was used as a control (the CK sample). After 35 min, both tubes of fig wasps were collected and immediately frozen in liquid nitrogen, and stored at $-80{ }^{\circ} \mathrm{C}$ until further processing. Total RNA was extracted from the adult wasps using Trizol reagent (Invitrogen, Carlsbad, CA, USA) according to the manufacturer's instructions. The quantity of RNA in the samples was assessed using $1.1 \%$ agarose gel electrophoresis and a NanoDrop 2000 spectrophotometer (NanoDrop, Wilmington, DE, USA).

For each sample, a messenger RNA-Seq library was constructed using an Illumina TruSeq ${ }^{\mathrm{TM}}$ RNA Sample Preparation Kit (Illumina, San Diego, USA) following the manufacturer's recommendations. The isolation of messenger RNA (mRNA), fragment interruption, complementary DNA (cDNA) synthesis, adaptor ligation, PCR amplification, and RNA-Seq were performed by Novogene Bioinformatics Technology Co. Ltd. (Beijing China). Poly-A mRNA was isolated using poly-Toligoattached magnetic beads and then broken into small pieces which were used as a template for synthesis of the first- and second-strand cDNA. A single "A" base was ligated to the short fragments after being purified using the AMPure XP system (Beckman Coulter, Beverly, USA), preparing them for ligation to the sequencing adapters. Fragments (200 bp) were then quantified using the high sensitivity DNA assay on the Agilent Bioanalyzer 2100 system and selected for PCR amplification as sequencing templates. Finally, the RNA-Seq library was sequenced on an Illumina Hiseq 2000 platform.

\subsection{Sequence cleaning, assembly, and contig annotation}

To obtain high-quality read data for de novo assembly, reads containing adaptor sequences, poly-N sequences, or quality scores (Q-values) lower than 20 were removed from the raw RNASeq data using Perl scripts from Novogene Bioinformatics Technology Co. Ltd. (Beijing 
China). At the same time, Q20, Q30, GC-content, and sequence duplication levels of the clean data were calculated. All downstream analyses were based on the cleaned, high-quality data.

The cleaned reads were extended into contigs through the overlap between the sequences using the Trinity de novo assembly software (Grabherr et al., 2011) with min_kmer_cov set to 2 and all other parameters set to default. Subsequently, the contigs were linked into transcripts and the transcripts clustered based on nucleotide sequence identity using the same software. The longest transcripts in the cluster units were regarded as unigenes to eliminate redundant sequences and then were combined to produce the final assembly used for annotation.

\subsection{Differential expression analysis}

In order to estimate the relative expression levels of the unigenes and provide more basal information for genetic and genomics studies on the molecular mechanisms related to $\mathrm{V}$. javana response to VOCs, we mapped the RNA-Seq data back to the transcriptome assemblies for each sample using RSEM (RNA-Seq by expectation-maximization; Li and Dewey, 2011) and BOWTIE (mismatch 2; parameters set default; Langmead et al., 2009). RSEM/BOWTIE mapping was implemented using scripts packaged with the Trinity pipeline. The significance of differences in gene expression between the treated and untreated wasps was determined using the R package DESeq (Wang et al., 2010). Statistical significance (p value) was adjusted using the q value obtained from the false discovery rate (Storey and Tibshirani, 2003), with a q value $<0.005$ and $\mid \log 2$ (foldchange) $\mid>2$ set as the threshold for significantly differential expression.

\subsection{GO and KEGG enrichment analysis of differentially expressed transcripts}

Gene Ontology (GO) enrichment analysis of the differentially expressed genes (DEGs) was implemented by the GOseqR package based on the Wallenius non-central hyper-geometric distribution (Young et al., 2010), which can be adjusted for gene length bias in DEGs. KEGG (Kanehisa et al., 2008) is a database resource for understanding highlevel functions and utilities of biological systems, such as the cell, the organism and the ecosystem, from molecular-level information, especially from molecular-level datasets generated by genome sequencing and other high-throughput experimental technologies (http://www. genome.jp/kegg/). We used the KOBAS software (Mao et al., 2005) to test the statistical enrichment of differential expression genes in KEGG pathways.

\subsection{Confirmation of the VOCs-responsive expression profiles by qRT-PCR}

The differential expression of genes identified by the above described method was validated using quantitative real-time PCR (qPCR). The real-time PCR was performed with the SYBR ${ }^{\circledR}$ Premix ExTaq TM (TaKaRa, Dalian, China) on the ABI 7500 Real-Time PCR System (Applied Biosystems, Foster City, CA, USA). The beta-actin gene was used as a reference control. The reaction was performed using the following conditions: denaturation at $95^{\circ} \mathrm{C}$ for $30 \mathrm{~s}$, followed by 40 cycles of amplification $\left(95{ }^{\circ} \mathrm{C}\right.$ for $10 \mathrm{~s}, 61^{\circ} \mathrm{C}$ for $20 \mathrm{~s}$ ). Each plate was repeated three 
times in independent runs for all reference and selected genes. Gene expression was evaluated by the $2^{-\Delta \Delta \mathrm{Ct}}$ method (Livak and Schmittgen, 2001).

\subsection{Statistical analysis}

For each sample, three technical replicates of the qRT-PCR assay were used with a minimum of three biological replicates. Results were expressed as means \pm standard deviation (SD) of the number of experiments. A Student's t-test was used to test if there was a significant difference in expression amount of a selected gene between different treatments.

\section{Results and discussion}

\subsection{Sequencing and de novo transcriptome assembly}

To record the variation in the Valisia javana transcriptome expression profile after exposure to volatile organic compounds (VOCs), two non-normalized libraries were constructed using VOC-treated fig wasps and control fig wasps with approximately $6.47 \mathrm{Gbp}$ and $6.48 \mathrm{Gbp}$ high quality reads respectively.

In total, 90,682,630 Illumina PE raw reads were generated (Table 1). After removing adaptor sequences, ambiguous nucleotides and low-quality sequences, there were 86,354,304 clean reads remaining. Assembly of clean reads resulted in 58,192 unigenes in the range of 20149,549 bp with a N50 length of 2711 bp (Fig. 1).

\subsection{Sequence annotation}

After eliminating repeated and short-length sequences, 58,192 nonredundant unigenes were subjected to seven public databases (Table 2) for similarity searching. Analyses showed that 12,805 unigenes $(22 \%)$ had significant matches in the $\mathrm{Nr}$ database, and $9900(17.01 \%)$ in the Nt database. Our results also showed that 10,217 (17.55\%) of the nonredundant unigenes were similar to known genes in Swiss-Prot database. In total, there were 17,417 unigenes (29.93\%) successfully annotated in at least one of the $\mathrm{Nr}$, Nt, the Swiss-Prot, KEGG, GO, COG and Pfam databases, with 3689 unigenes $(6.33 \%)$ in all seven databases.

Table 1. Summary of sequences analysis. CK: Control. SM: Treated xx. Q20: The percentage of bases with a Phred value > 20. Q30: The percentage of bases with a Phred value > 30 .

\begin{tabular}{llllllll}
\hline Sample & Raw Reads & Clean Reads & Clean Bases & Error $(\%)$ & Q20 $(\%)$ & Q30 $(\%)$ & $\begin{array}{l}\text { GC } \\
(\%)\end{array}$ \\
\hline CK & 45223494 & 43121846 & $6.47 \mathrm{G}$ & 0.02 & 95.8 & 90.56 & 39.67 \\
SM & 45459136 & 43232458 & $6.48 \mathrm{G}$ & 0.02 & 95.55 & 90.12 & 39.18 \\
Total & 90682630 & 86354304 & 12.95 & & & &
\end{tabular}




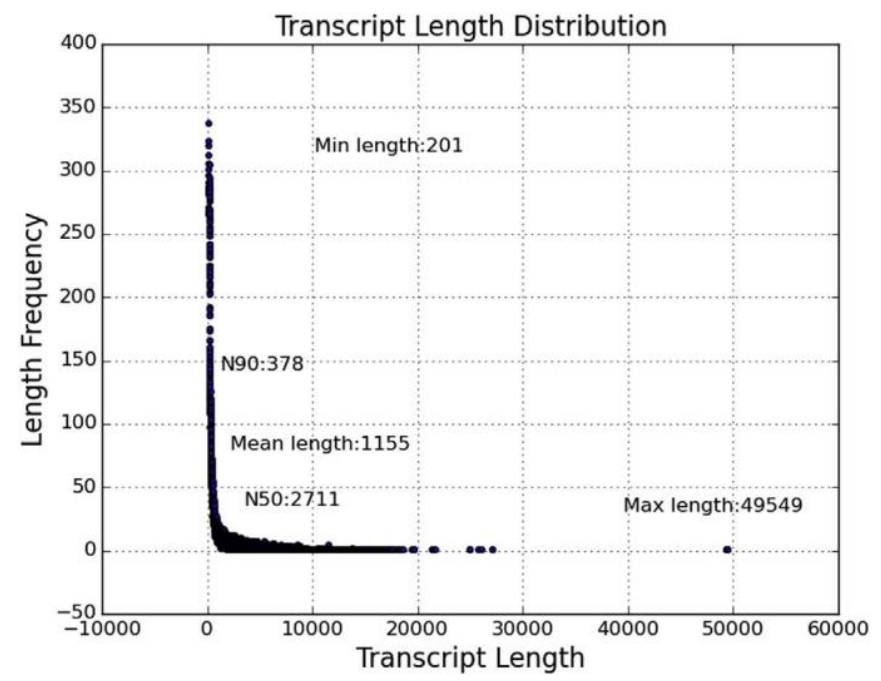

Fig. 1. Length distribution of assembled unigenes.

Table 2 BLAST analysis of non-redundant unigenes against public databases.

\begin{tabular}{lll}
\hline & $\begin{array}{l}\text { Number of } \\
\text { Unigenes }\end{array}$ & $\begin{array}{l}\text { Percentage } \\
(\%)\end{array}$ \\
\hline Annotated in NR & 12805 & 22 \\
Annotated in NT & 9900 & 17.01 \\
Annotated in KO & 6404 & 11 \\
Annotated in & 10217 & 17.55 \\
SwissProt & & \\
Annotated in PFAM & 12027 & 20.66 \\
$\begin{array}{l}\text { Annotated in GO } \\
\text { Annotated in KOG }\end{array}$ & 12086 & 20.76 \\
Annotated in all & 3764 & 13.34 \\
Databases & & 6.33 \\
Annotated in at least \\
one Database
\end{tabular}

Using Gene Ontology (GO), an international standardized gene functional classification system, 12,086 non-redundant unigenes were classified into three major functional ontologies (biological process, cellular component and molecular function) (Fig. 2). For biological processes, dominant subcategories were 'cellular processes' (6830) and 'metabolic processes' (6018). In the category of cellular component, 'cell' (3828), 'cell part' (3828) and 'macromolecular complex' (2540) were highly represented. Among molecular function terms, 'binding' (6525) and 'catalytic activity' (4726) were most represented, followed by 'transporter 
activity' (978). However, within each of the three categories, only a few genes were assigned to the subcategories of 'cell aggregation', 'nucleoid' and 'metallochaperone activity'.

In addition, all unigenes were subjected to a search in the COG database for functional prediction and classification. 7764 non-redundant unigenes (Table 2) were subdivided into 26 COG classifications (Fig. 3). Among them, the cluster of 'General function prediction only' (1308) was the largest group, followed by 'Signal transduction mechanisms' (1172), 'Posttranslational modification, protein turnover, chaperones' (810), 'Translation, ribosomal structure and biogenesis' (548) and 'Transcription' (489). Only a few unigenes were assigned to 'un-named protein' (2) and 'cell motility' (13).

The unigene metabolic pathway analysis was also conducted using the KEGG annotation system. According to KEGG, 6404 unigenes (Table 2) were assigned to 32 pathways (Fig. 4). The pathways involving the largest number of unique transcripts were 'Signal transduction' (875), followed by 'Translation' (591), whereas 'Biosynthesis of other secondary metabolites' (9) was the smallest group.

\subsection{GO and KEGG enrichment analysis of differently expressed genes}

To better survey the molecular aspects of fig wasp response to exposure to VOCs, it is necessary to detect the functional distribution of differentially expressed genes (DEGs) in V. javana via comparing its transcriptomes in response to different treatments.

GO analysis was conducted for the up-regulated DEGs in the SM sample. The GO enrichment of up-regulated DEGs is shown in Supplementary Table S1. In the cell components category, the top three enriched terms were "outer membrane-bounded periplasmic space", "periplasmic space" and "envelope". In the biological process category, the top three enriched terms were "pyruvate transport", "mitochondrial pyruvate transport" and "viral genome packaging". In the molecular function category, the top three enriched terms were "hyaluronic acid binding", "glycosaminoglycan binding" and "serine-type endopeptidase activity".

The GO enrichment of down-regulated DEGs in the SM sample s shown in Supplementary Table S2. For the down-regulated DEGs in the SM compared to the CK sample, "clathrin adaptor complex", "AP-type membrane coat adaptor complex" and "clathrin coat" were the topthree cellular components enriched by the down-regulated DEGs. In the molecular function category, the most enriched term was "odorant binding". 


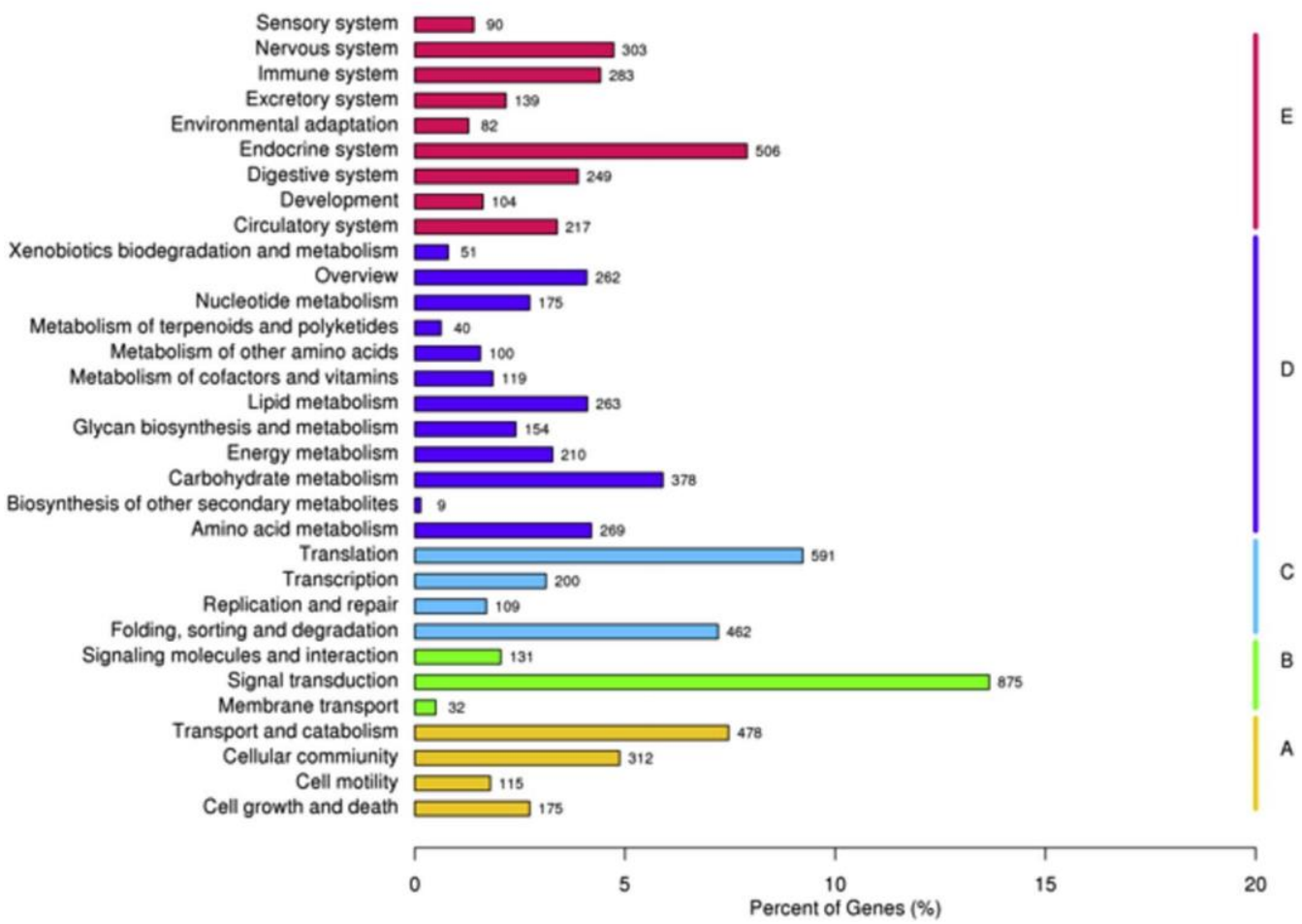

Fig. 4. KEGG annotation of putative proteins.

The correspondence between biological behavior and DEGs is complicated because the molecular response of fig wasps to VOCs involves a combination of various intra-cellular metabolic pathways. KEGG pathway enrichment analysis for DEGs also revealed the complicated pathways related to the fig wasp response to VOCs. "Endocrine and other factorregulated calcium reabsorption", "Synaptic vesicle cycle", "RNA degradation" and "Endocytosis" were the top-four enriched pathways $(q \leq 0.05)$ for the DEGs in the SM sample compared with the CK sample The KEGG pathway enrichment of DEGs in the SM is shown in Supplementary Table S3.

3.4. Olfactory nervous system related genes among the DEGs

Chemoreception by fig wasps plays an important role in the interaction between figs and their pollinating wasps, and odorant-binding proteins (OBP) function in the first step of odorant detection (Pelosi and Maida, 1990). In the GO enrichment up and down regulated DEGs, there were two unigenes annotated as "odorant binding", an important odorant binding protein(OBP) gene - c1583_g1 with a 9.87-fold increased and c19239_g2-with -2.06-fold decreased expression The unigene c9496_g1 with a -9.98-fold expression was annotated as "clathrin adaptor complex", "AP-type membrane coat adaptor complex" and "clathrin coat", and identified as a putative AP2a protein in the synaptic vesicle cycle pathway (Fig. 5). Using the 7 public databases, we found 8 DEGs which were putative OBP encoding genes by Blast analysis. 
Among them, the expression difference of 6 DGEs varied less than twofold. The sequences of these putative OBP genes are listed in Table S4. It should be noted that because OBP family members are highly divergent in insects, and their overall sequence identity is as low as $16.71 \%$ (Vieira and Rozas, 2011a,b), some OBP genes may not have been detected by blast analysis. The results confirm that VOCs treatment influences the olfactory system of fig wasps.

Odorant-binding proteins (OBPs) are extracellular proteins found in insect chemosensilla, where they participate in the sensing of odors, tastes, and pheromones. OBPs are present at high concentrations in the lymph fluid of the antennal sensilla and are further subdivided into general

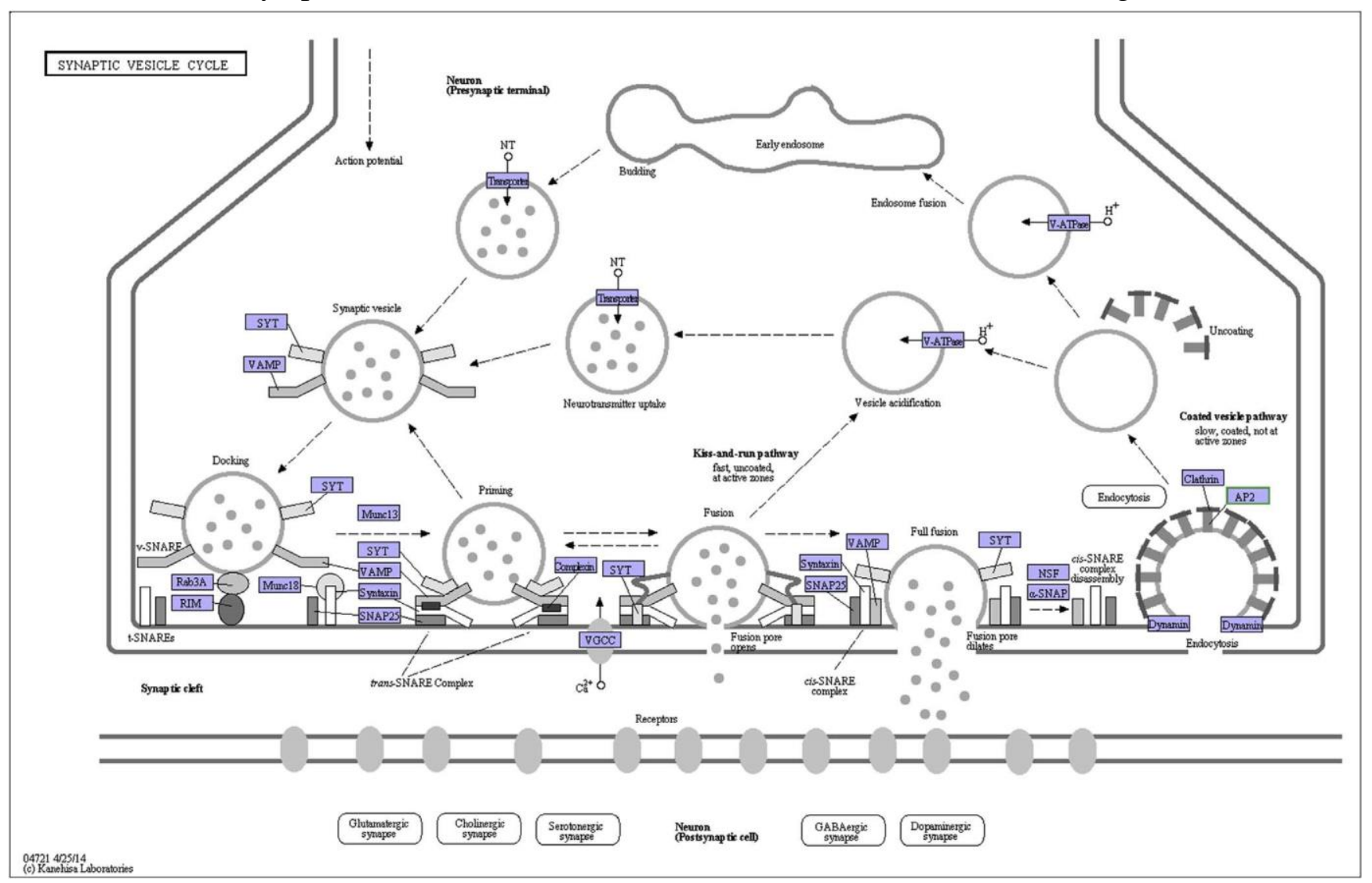

Fig. 5. Synaptic vesicle cycle pathway.

odorant binding proteins (GOBPs) (Vogt and Lerner, 1989; Vogt et al., 2004), pheromone binding protein (PBPs) (Vogt and Lerner, 1989) and other types of odorant binding proteins, such as ABPX, etc. (Krieger et al., 1996). PBPs are thought primarily to bind and transport the sex pheromones in insects. GOBPs are postulated to be involved in the reception of volatile chemicals produced by plants. In this study, the unigene c1583_g1 of V. javana was presumed to be a general odorantbinding protein 56d-like gene by blastx analysis in NCBI, with $43 \%$ identity the protein Copidosoma floridanum (Hymenoptera, Encyrtidae). C1583_g1 was found to be strongly up-regulated after VOCs treatment, suggesting that this GOBP gene might be involved in VOC perception in V. javana. On the other hand, the unigene c19239_g2 was 
inferred to be a pheromone-binding protein-related protein 6-like gene and its downregulation suggests that the fig wasps might concentrate VOC perception by reducing sex pheromone binding activity when treated with receptive fig VOCs.

Although a large number of OBP genes have been identified in insect genomes, their molecular functions and biological roles have been clarified in limited cases. In recent years, some studies have reported that there are interactions between different OBPs to perform specific functions (Fan et al., 2011). OBPs can be expressed in antennae but also in the head, thorax, abdomen, feet, wings and other tissues (Krieger and Breer, 1999; Li et al., 2008; Vogel et al., 2010; Hua et al., 2012; Yin et al., 2012) suggesting that the function of OBPs may not be limited to olfaction. In this study, the exact functions of the differentially expressed OBP genes in the perception of VOCs are unknown. We infer that they might also regulate the chemoreception of VOCs positively or negatively by interacting with each other. Because the experimental samples came from the whole adult fig wasps, the OBP genes detected in this study might not be exclusively expressed in antennae, and they could also be expressed in other tissues. Therefore, the localization of these OBPs and what their functions are remain to be further investigated.

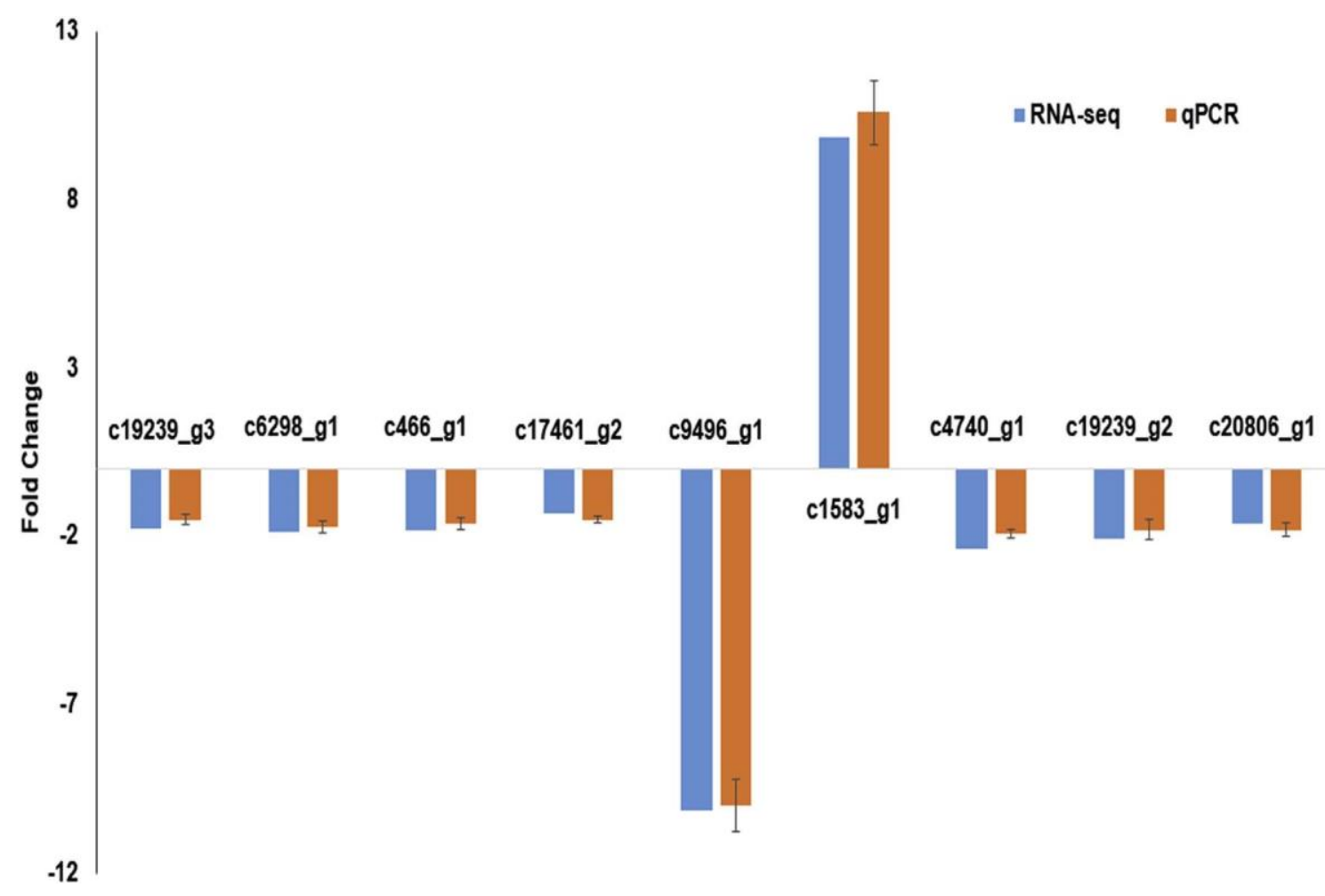

Fig. 6. Comparison of the expressions of RNA-Seq and qRT-PCR results. The transcript expression levels of the selected genes were each normalized to that of the $\beta$-actin gene. 


\subsection{Validation of differentially expressed genes using qRT-PCR}

To confirm the reliability of the RNA-Seq data, the transcription levels of one AP2a unigene and eight OBP unigenes were examined using real-time quantitative PCR (Fig. 6). All the primers designed for V. javana are shown in Supplementary Table S5. All the primers were used for PCR-amplification in the cDNA of the CK and SM samples, and genomic DNA of V. javana. Among the one AP2A unigene and eight OBP unigenes, only two genes exhibited an over 2 fold higher expression in response to VOCs, a result which was consistent with the RNASeq data. So the RNA-Seq data were credible.

\section{Conclusion}

In this study, we characterized the transcriptomes from VOC-treated (SM sample) and control samples (CK sample) of the pollinating fig wasp, Valisia javana, obtaining an unprecedented amount of genomic resources for this species. Twenty nine Differentially-expressed genes were identified between fig wasps that had been exposed and that had not been exposed to receptive fig VOCs. Among these, Odor Binding Proteins were present. This suggests a modification of the insect's olfactory signaling pathway in response to the VOC treatment. This may imply, for instance, that tests of fig wasp response to VOCs in olfactometers and also GC-EAD tests of antennal responses may need to investigate the effect of preconditioning of the wasps. The olfactory signaling pathway may vary between the moment the wasp first senses receptive fig odor and the moment the wasps takes the decision to land on a supposedly receptive fig. The results presented here are preliminary but they open such unexpected avenues of research.

\section{Acknowledgements}

This work was supported by the Key Program of National Natural Science Foundation of China (31630008), National Natural Science Foundation of China (31670395; 31370409), and Natural Science Foundation of Guangdong Province (S2013020012814).

Supplementary data related to this article can be found at http://dx. doi.org/10.1016/j.actao.2018.01.003.

\section{References}

Beenakkers, A.M.T., van der Horst, D.J., van Marrewijk, W.J.A., 1984. Insect flight muscle metabolism. Insect Biochem. 14, 243-260.

Benton, R., Vannice, K.S., Gomez-Diaz, C., Vosshall, L.B., 2009. Variant ionotropic glutamate receptors as chemosensory receptors in Drosophila. Cell 136, 149-162.

Berg, C.C., 2003. Flora Malesiana precursor for the treatment of Moraceae 1: the main subdivision of Ficus: the subgenera. Blumea 48, 167-178.

Cook, J.M., Segar, S.T., 2010. Speciation in fig wasps. Ecol. Entomol. 35 (Suppl. 1), 54-66. 
Cruaud, A., Jabbour-Zahab, R., Genson, G., et al., 2010. Laying the foundations for a new classification of Agaonidae (Hymenoptera: Chalcidoidea), a multilocus phylogenetic approach. Cladistics 26, 359-387.

de Bruyne, M., Baker, T.C., 2008. Odor detection in insects: volatile codes. J. Chem. Ecol. 34, 882-897.

Fan, J., Francis, F., Liu, Y., Chen, J.L., Cheng, D.F., 2011. An overview of odorant-binding protein functions in insect peripheral olfactory reception. Genet. Mol. Res. 10, 3056-3069.

Gibernau, M., Hossaert-McKey, M., Frey, J., Kjellberg, F., 1998. Are olfactory signals sufficient to attract fig pollinators? Ecoscience 5, 306-311.

Grabherr, M.G., Haas, B.J., Yassour, M., et al., 2011. Full-length transcriptome assembly

from RNA-Seq data without a reference genome. Nat. Biotechnol. 29, 644-652.

Grison-Pigé, L., Bessière, J.M., Hossaert-McKey, M., 2002. Specific attraction of figpollinating wasps: role of volatile compounds released by tropical figs. J. Chem. Ecol. 28, 283-295.

Hossaert-Mckey, M., Gibernau, M., Frey, J.E., 1994. Chemosensory attraction of fig wasps to substances produced by receptive figs. Entomol. Exp. Appl. 70, 185-191.

Hossaert-Mckey, M., Soler, C., Schatz, B., Proffit, M., 2010. Floral scents: their roles in nursery pollination mutualisms. Chemoecology 20, 75-88.

Hua, J.F., Zhang, S., Cui, J.J., et al., 2012. Identification and binding characterization of three odorant binding proteins and one chemosensory protein from Apolygus lucorum (MeyerDur). J. Chem. Ecol. 38, 1163-1170.

Kanehisa, M., et al., 2008. KEGG for linking genomes to life and the environment. Nucleic Acids Res. 36, D480-D484.

Kaupp, U.B., 2010. Olfactory signalling in vertebrates and insects: differences and commonalities. Nat. Rev. Neurosci. 11, 188-200.

Kwon, J.Y., Dahanukar, A., Weiss, L.A., Carlson, J.R., 2007. The molecular basis of CO2 reception in Drosophila. Proc. Natl. Acad. Sci. U.S.A. 104, 3574-3578.

Krieger, J., Breer, H., 1999. Olfactory reception in invertebrates. Science 286, 720-728.

Krieger, J., von Nickisch-Rosenegk, E., Mameli, M., Pelosi, P., Breer, H., 1996. Binding proteins from the antennae of Bombyx mori. Insect Biochem. Mol. Biol. 26, 297-307.

Langmead, B., Trapnell, C., Pop, M., Salzberg, S.L., 2009. Ultrafast and memory-efficient alignment of short DNA sequences to the human genome. Genome Biol. 10, R25.

Li, B., Dewey, C.N., 2011. RSEM: accurate transcript quantification from RNA-Seq data with or without a reference genome. BMC Bioinf. 12, 323.

Li, S., Picimbon, J.F., Ji, S., et al., 2008. Multiple functions of an odorant-binding protein in the mosquito Aedes aegypti. Biochem. Bioph.Res. Co 372, 464-468.

Livak, K.J., Schmittgen, T.D., 2001. Analysis of relative gene expression data using realtime quantitative PCR and the 2 (-Delta Delta C(T)) Method. Methods 25, 402-408.

Mao, X., Cai, T., Olyarchuk, J.G., Wei, L., 2005. Automated genome annotation and pathway identification using the KEGG Orthology (KO) as a controlled vocabulary.

Bioinformatics 21, 3787-3793. 
Pelosi, P., Calvello, M., Ban, L., 2005. Diversity of odorant-binding proteins and chemosensory proteins in insects. Chem. Senses 30 (Suppl. 1), i291-i292.

Pelosi, P., Maida, R., 1990. Odorant-binding proteins in vertebrates and insects: similarities and possible common function. Chem. Senses 15, 205-215.

Raguso, R.A., 2008. Start making scents: the challenge of integrating chemistry into pollination ecology. Entomol. Exp. Appl. 128, 196-207.

Sanchez-Gracia, A., Vieira, F.G., Rozas, J., 2009. Molecular evolution of the major chemosensory gene families in insects. Heredity 103, 208-216.

Snyder, S.H., Sklar, P.B., Pevsner, J., 1988. Molecular mechanisms of olfaction. J. Biol. Chem. 263, 13971-13974.

Soler, C., Hossaert-McKey, M., Buatois, B., Bessière, J.M., Schatz, B., Proffit, M., 2011. Geographic variation of floral scent in a highly specialized pollination mutualism. Phytochemistry 72, 74-81.

Storey, J.D., Tibshirani, R., 2003. Statistical significance for genome wide studies. Proc. Natl. Acad. Sci. Unit. States Am. 100, 9440-9445.

Touhara, K., Vosshall, L.B., 2009. Sensing odorants and pheromones with chemosensory receptors. Annu. Rev. Physiol. 71, 307-332.

van Noort, S., Compton, S.G., 1996. Convergent evolution of Agaoninae and Sycoecinae (Agaonidae, Chalcidoidea) head shape in response to the constraints of host fig morphology. J. Biogeogr. 23, 415-424.

Vieira, F.G., Rozas, J., 2011a. Comparative genomics of the odorant-binding and chemosensory protein gene families across the Arthropoda: origin and evolutionary history of the chemosensory system. Genome Biol. Evol. 3, 476-490.

Vieira, F.G., Rozas, J., 2011b. Comparative genomics of the odorant-binding and chemosensory protein gene families across the Arthropoda: origin and evolutionary history of the chemosensory system. Genome Biol. Evol. 3, 476-490.

Vogel, H., Heidel, A.J., Heckel, D.G., et al., 2010. Transcriptome analysis of the sex pheromone gland of the noctuid moth Heliothis virescens. BMC Genomics 1, 29.

Vogt, R., Lerner, M., 1989. Two groups of odorant binding proteins in insects suggest specific and general olfactory pathways. Soc. Neurosci. Abstr. 15, 1290-1296.

Vogt, R.G., Prestwich, G.D., Lerner, M.R., 2004. Odorantbinding- protein subfamilies associate with distinct classes of olfactory receptor neurons in insects. J. Neurobiol. 22, 7484.

Vosshall, L.B., Stocker, R.F., 2007. Molecular architecture of smell and taste in Drosophila. Annu. Rev. Neurosci. 30, 505-533.

Wang, L., Feng, Z., Wang, X., Wang, X., Zhang, X., 2010. DEGseq: an R package for identifying differentially expressed genes from RNA-seq data. Bioinformatics 26, 136-138.

Wang, Z., Gerstein, M., a Snyder, M., 2009. RNA-Seq: a revolutionary tool for transcriptomics. Nat. Rev. Genet. 10, 57-63.

Wang, N., Wang, N.X., Niu, L.M., Bian, S.N., Xiao, J.H., et al., 2014. Odorant-binding protein (OBP) genes affect host specificity in a fig-pollinator mutualistic system. 
Insect Mol. Bio 23, 621-631.

Weiblen, G.D., 2002. How to be a fig wasp. Annu. Rev. Entomol. 47, 299-330.

Xiao, J.H., Yue, Z., Jia, L.Y., Yang, X.H., Niu, L.H., et al., 2013. Obligate mutualism within a host drives the extreme specialization of a fig wasp genome. Genome Biol. 14, R141.

Yin, J., Feng, H., Sun, H., et al., 2012. Functional analysis of general odorant binding protein 2 from the Meadow Moth, Loxostege sticticalis L. (Lepidoptera: Pyralidae).

PLoS One 7, e33589.

Young, M.D., Wakefield, M.J., Smyth, G.K., Oshlack, A., 2010. Gene ontology analysis for RNA-seq: accounting for selection bias. Genome Biol. 11, R14.

Yu, H., Compton, S.G., 2012. Moving your sons to safety: galls containing male fig wasps expand into the centre of figs, away from enemies. PLoS One 7, e30833.

Zhou, Z.K., Gilbert, M.G., 2003. Ficus. In: Flora of China (English Edition) 5. Science Press, Beijing, China \& Missouri Botanical Garden Press, St. Louis, pp. 37-73 Flora of China Editorial Committee. 